\title{
CUIDADOS DE ENFERMAGEM EM RELAÇÃO A MULHER VÍTIMA DE VIOLÊNCIA DOMÉSTICA NA ATENÇÃ̃O PRIMÁRIA À SAÚDE
}

\section{NURSING CARES IN RELATION TO WOMEN VICTIMS OF DOMESTIC VIOLENCE IN PRIMARY HEALTH CARE}

\author{
Jhuliano Silva Ramos de Souza ${ }^{1} *$ Patrícia Lemos Magalhães $^{2} *$ Sueli de Carvalho Vilella $^{3} *$ \\ Zélia Marilda Rodrigues Resck ${ }^{4}$
}

\begin{abstract}
RESUMO
Objetivo: refletir sobre cuidados de enfermagem em relação a mulher vítima de violência doméstica na Atenção Primária à Saúde. Método: Trata-se de um estudo teórico-reflexivo, com a coleta de dados em março de 2021, por meio das bases de dados: Literatura Latino-Americana e do Caribe em Ciências da Saúde (LILACS), National Library of Medicine (PUBMED) e Scientific Electronic Library Online (SCIELO). Foram selecionados seis estudos dos últimos dez anos (20102020), utilizando a análise de conteúdo para a categorização dos artigos. Resultados: Emergiu duas categorias sobre o cuidado de enfermagem a mulher vítima de violência na atenção básica e capacitação dos profissionais de enfermagem frente no reconhecimento da violência doméstica. Considerações finais: As dificuldades que os profissionais de enfermagem têm ao cuidado a mulheres em situações de violência, estão relacionadas a falta de formação qualificada, treinamento e capacitação, trazendo sentimento de impotência e despreparo perante as situações vivenciadas na prática clínica, como o encaminhamento aos serviços de referência e a familiaridade com a ficha de notificação compulsória.
\end{abstract}

Palavras-chave: Equipe de Enfermagem; Atenção Primária à Saúde; Violência contra a Mulher.

\begin{abstract}
Objective: to reflect on nursing care in relation to women victims of domestic violence in Primary Health Care. Method: This is a theoretical-reflective study, with data collection in March 2021, through the databases data: Latin American and Caribbean Literature in Health Sciences (LILACS), National Library of Medicine (PUBMED) and Scientific Electronic Library Online (SCIELO). Six studies from the last ten years (2010-2020) were selected, using content analysis for the categorization of articles. Results: Two categories emerged on nursing care for women victims of violence in primary care and training of nursing professionals in the recognition of domestic violence. Final considerations: The difficulties that nursing professionals have in caring for women in situations of violence are related to the lack of qualified education, training and qualification, bringing a feeling of helplessness and unpreparedness in the situations experienced in clinical practice, such as referral to patients. reference services and familiarity with the compulsory notification form.
\end{abstract}

Keywords: Nursing, Team; Violence Against Women; Primary Health Care.

\footnotetext{
${ }^{1}$ Enfermeiro, Mestre e Doutorando em Enfermagem pelo Programa de Pós-Graduação em Enfermagem da Universidade Federal de Alfenas (UNIFAL-MG). E-mail: jhulianoramoz@ hotmail.com ORCID: https://orcid.org/0000-0002-4338-4433

${ }^{2}$ Enfermeira, Mestrando em Enfermagem pelo Programa de Pós-Graduação em Enfermagem da Universidade Federal de Alfenas (UNIFAL-MG). E-mail: patilemos37@ gmail.com ORCID: https://orcid.org/0000-0001-5974-5451

${ }^{3}$ Enfermeira, Doutora em Ciências pela Escola de Enfermagem de Ribeirão Preto da Universidade de São Paulo (EERP/USP). Docente do Programa de Pós-Graduação em Enfermagem da Universidade Federal de Alfenas (UNIFAL-MG). E-mail: suelicvilela@gmail.com ORCID: https://orcid.org/0000-0003-3034-3904

${ }^{4}$ Enfermeira, Pós-doutorado em Ciências pela Escola de Enfermagem de Ribeirão Preto da Universidade de São Paulo (EERP/USP). Docente do Programa de Pós-Graduação em Enfermagem da Universidade Federal de Alfenas (UNIFAL-MG). E-mail: zmrresck57@gmail.com ORCID: https://orcid.org/0000-0002-3752-8381
} 


\section{INTRODUÇÃO}

A Organização Mundial da Saúde define a violência como o uso intencional da força física, do poder ou ameaça contra a si, a outrem, a um grupo ou até mesmo a seus pares, resultando danos não só de natureza física (lesões corporais), como psicológica e até a morte ${ }^{(1)}$. Existe diversas formas de violência que as pessoas podem ser afetadas bem como pode acontecer em diversos estratos, e um deles é a violência contra a mulher, essa é considerada uma violação dos direitos humanos ao gênero feminino, independente da étnica, classe social, religião, questões culturais, fatores econômicos, dentre outros, podendo trazer diversas consequências traumáticas a vida dessa mulher ${ }^{(2)}$.

Em 2018, 4.519 mulheres morreram em decorrência da violência contra a mulher no Brasil, a maioria sendo negras que residiam nas regiões norte e nordeste, destacando a causa morte por feminicídio em que os autores desse crime eram seus parceiros/companheiros que viviam em seu convívio familiar ${ }^{(3)}$.

Conforme a Lei $\mathrm{n}^{\mathrm{o}} 11.340$, de 7 de agosto de 2006, conhecida como Lei Maria da Penha que protege mulheres que sofrem/sofreram qualquer tipo de violência no Brasil, a mesma define que a violência a mulher é "qualquer ação ou omissão que lhe cause morte, lesão, sofrimento físico, sexual ou psicológico e dano moral ou patrimonial" trazendo diversas classificações quanto as tipologias da violência, como a violência doméstica e a intrafamiliar ${ }^{(4)}$.

No que se refere a violência no âmbito doméstico, é todo ambiente de convivência de pessoas que residem no mesmo local sem ter vínculo familiar de cunho biológico. Em relação ao intrafamiliar entende-se como um grupo de pessoas que tem algum parentesco, seja de cunho biológico ou não que estão interligados por alguma razão, afinidade ou vontade (4). Os termos tanto da violência doméstica como da intrafamiliar apresentam semelhanças entre seus conceitos, porém apresentando pequenas distinções entre suas definições ${ }^{(5)}$.

Em vista desse fenômeno de natureza criminal, o mesmo se tornou um sério problema de saúde pública. Neste sentido, os profissionais de saúde, em especial, os da enfermagem devem estar preparados para o atendimento a essas mulheres em situações de violência nos serviços de saúde, como na Atenção Primária à Saúde (APS) uma vez que essa modalidade de serviço é a porta de entrada no atendimento de mulheres em situações de violência $^{(6)}$.

A atuação da equipe de enfermagem na APS no atendimento de mulheres vítimas de violência doméstica está relacionada ao acolhimento, empatia, escuta terapêutica, criação de vínculo e de confiança entre enfermeiro-paciente para a criação de um bom relacionamento interpessoal, pois muitas mulheres têm resistência de compartilhar 
sobre a violência que sofre em seu convívio familiar $^{(7)}$.

Os profissionais da saúde que forem atender essas vítimas, devem possuir competências e habilidades para o enfretamento da violência. No entanto, ainda há divergências sobre identificar quais papéis que os mesmos deverão ter na identificação de sinais físicos da violência e no encaminhamento aos serviços de segurança pública, justiça e serviço social. A atuação dos profissionais demostra que eles ignoram certas características da epidemiologia da violência contra a mulher, especialmente com serviço pré-natal ${ }^{(8)}$.

Quanto aos profissionais de enfermagem, ainda falta de treinamento no cuidado a pessoas em situações de violência, sendo uma das principais lacunas é a falta de formação, treinamento e capacitação dos mesmos, trazendo sentimento de impotência perante as situações vivenciadas e os processos de enfermagem que deverão ser aplicados na prática clínica, bem como no encaminhamento aos serviços de referência (7).

Talvez pela falta de capacitação para o cuidado com a mulher os profissionais de enfermagem compreendem que a violência doméstica é apenas identificada pelos sinais físicos, descartando outros indicadores que são extremamente presentes nas vítimas de violência contra a mulher ${ }^{(9)}$, podendo estar inserida em diversos contextos da vida, dentre eles o privado, doméstica e familiar, fazendose necessário que o acolhimento e o atendimento a essas vítimas seja efetivo, proativo, humanizado e ético perante aos desafios e problemas que o setores saúde acabam enfrentando no seu cotidiano ${ }^{(10)}$.

Diante dessa contextualização o objetivo desse estudo foi refletir sobre cuidados de enfermagem em relação a mulher vítima de violência doméstica na APS.

\section{MÉTODO}

Trata-se de um estudo teóricoreflexivo sobre cuidados de enfermagem em relação a mulher como vítima de violência doméstica na APS. A coleta de dados ocorreu em março de 2021, por meio das bases/portais de dados eletrônicas da Biblioteca Virtual em Saúde (BVS): Literatura Latino-Americana e do Caribe em Ciências da Saúde (LILACS), e Medical Literature and Retrivial Sistem onLine (MEDLINE) e Scientific Electronic Library Online (SCIELO).

Foram utilizados os Descritores em Ciências da Saúde (DeCS) e do Medical Subject Headings da U.S.National Library of Medicine (Mesh): Equipe de Enfermagem $A N D$ Violência contra a Mulher; Atenção Primária à Saúde $A N D$ Violência contra a Mulher; Nursing, Team AND Violence Against Women; Primary Health Care AND Violence Against Women; Grupo de Enfermería $A N D$ Violencia contra la Mujer; 
Atención Primaria de Salud AND Violencia contra la Mujer, nos idiomas português, inglês e espanhol nos últimos dez anos (20102020).

Os critérios de inclusão foram estudos que relacionam a atuação da enfermagem no cuidado às mulheres que sofrem violência na APS. Já os critérios de exclusão foram estudos que abordam sobre a violência contra a mulher sem a participação da enfermagem no cuidado e/ou relacionado a outros tipos de violência, como por exemplo, contra crianças, adolescentes e idosos.

Para análise dos estudos incluídos, foi utilizado a análise de conteúdo de Bardin (11) seguindo as três fases por ela indicadas 1) pré-análise, 2) exploração do material e 3) tratamento dos resultados, inferência e interpretação. Feito a categorização dos mesmos para a discussão reflexiva sobre a temática abordada.

\section{RESULTADOS}

A busca bibliográfica resultou em 703 estudos na primeira seleção feita pelas leituras de títulos e resumos, o qual foi feito a exclusão de artigos duplicados (14) e os que não atenderam os critérios de inclusão (668) sendo estudos relacionados a abordagem multiprofissional sobre a violência doméstica e/ou relacionado a outros tipos de violência.

Por meio da primeira seleção dos estudos, 21 estudos foram pré-selecionados, dentre os quais foram lidos na íntegra sendo 15 encontradas na MEDLINE, 5 na LILACS e 1 na SCIELO, não atenderam o objetivo de estudo por se tratar de temas relacionados a abordagem de vítimas de violência no âmbito hospitalar, forense, paliativo, psiquiátrico e saúde mental e de outros idiomas. Assim, a amostra resultou em seis artigos.

Diante da análise dos estudos selecionados, emergiram duas categorias para a discussão sobre: I) O cuidado de enfermagem a mulher vítima de violência na atenção básica; e II) Capacitação dos profissionais de enfermagem frente no reconhecimento da violência doméstica.

\section{DISCUSSÃO}

\section{I) O cuidado de enfermagem a mulher} vítima de violência na atenção básica

A APS é um ambiente que favorece na identificação de mulheres vítimas de violência doméstica por serem assistidas nesses serviços e por ser considerado um serviço de porta de entrada bem como pelo fato da estrutura do mesmo e do seu intercambio com a família e com o habitat da população ${ }^{(12)}$.

As características que ajudam na identificação das mulheres vítimas de violência são relacionadas à dependência econômica e afetiva em relação ao parceiro, medo do agressor, do julgamento externo e até mesmo da morte por conta das ameaças. 
Assim como, pelas lesões físicas, sinais de coerções, pelo aspecto psicológico e emocional que podem ser percebidos durante a consulta de enfermagem ou mesmo em momentos de realização de procedimentos específicos e em visitas domiciliares. Dessa forma, atenta-se que diferentes tipos de violência podem ser identificados, quanto a violência doméstica definida como violação dos direitos da mulher, podendo ser classificadas como física, verbal, moral e psicológica, sendo a física, identificada como uma das mais conhecidas ${ }^{(9)}$.

$\mathrm{O}$ cuidado a mulher vítima de violência doméstica envolve a assistência integral nos aspectos físicos e biológicos, psicológicos, sociais, legais. Identificar e compreender os sentimentos experenciados pelas vítimas de violência, além de lesões físicas, possibilitam que as intervenções sejam resolutivas. Para tanto, a comunicação e a escuta reflexiva são estratégias que marcam a eficiência no atendimento, em que os enfermeiros referem a existência de problemas entre a comunicação e a escuta frente ao cuidado a essas mulheres ${ }^{(13)}$. Outra vertente do cuidado é possibilitar o amparo, encorajamento, empoderamento para que a mulher se sinta protegida, respeitada e segura quando estiver buscando atendimento nos serviços de saúde, preservando assim sua saúde mental das mesmas ${ }^{(14)}$.

Por outro lado, o enfermeiro que atua na APS pode identificar as vítimas em relação as consequências deixadas pela violência uma vez que podem gerar traumas marcantes a essas vítimas, como por exemplo, os quais irão afetar seu estado biopsicossocial podendo ser precursor de sintomas depressivos, isolamento, medo e incapacidade de tomar decisões e outros.

No âmbito legal, os enfermeiros devem auxiliar as vítimas a buscarem seus direitos e proteção. Nesse sentido, há despreparo da equipe de enfermagem quanto ao conhecimento da legislação e da notificação compulsória, o que gera insegurança, medo e dificuldades em prestarem informações corretas no atendimento de pessoas em situações de violência ${ }^{(13)}$.

As relações interpessoais entre enfermeiros e mulheres vítimas de violência domesticas devem se fundamentadas na criação de vínculos para que consigam encorajá-las e emponderá-las sobre seus direitos e suas decisões frente a violência por parceiro íntimo, assim como favorece a sensação de confiança, crença no sigilo, a empatia, a confiança no profissional. Por outro lado, favorece ao profissional a emancipação dos conhecimentos técnicocientíficos, habilidades e competências para assistir mulheres em situações violentas ${ }^{(10)}$.

Em vista disso, os cuidados de enfermagem frente a mulher vítima de violência doméstica devem ser pautados na promoção, segurança, acolhimento, respeito, 
planejamento de ações estratégicas, políticas públicas e legislação vigente para a proteção e para prevenção das consequências que a violência acarreta na vida dessas mulheres. Como também ao acolhimento, a escuta qualificada, a criação do vínculo, ao encorajamento a denunciar, ao exame físico detalhado e na abordagem psicoemocional, em que os serviços de atenção básica devem estar preparados para receber/atender essas mulheres em situações de violência ${ }^{(9)}$.

Além disso, ainda permanece as falhas no atendimento por não terem familiaridades com a ficha de notificação compulsória, no acolhimento, no encaminhamento ao psicólogo, ao assistente social e a segurança pública, não havendo retorno e o acompanhamento de perto pelo enfermeiro que atua na atenção primária ${ }^{(12)}$.

Quanto ao conhecimento dos enfermeiros da APS a respeito da violência contra a mulher, observa-se que há necessidade de uma abordagem interdisciplinar nos serviços de segurança pública, social, jurídico e de educação ${ }^{(15)}$.

O conhecimento dos profissionais sobre o cuidado a mulher vítima de violência, é deficitário uma vez que esse não tem formação qualificada, desconhecem as características epidemiológicas da violência, não sabem qual conduta correta tomar frente aos casos, como também há a falta de um instrumento que norteie esse tipo de atendimento específico, o que dificultando o atendimento, diminui a sensibilidade em acolher a vítima e dificulta o encaminhamento para a segurança pública ${ }^{(16)}$.

É importante que os enfermeiros saibam identificar os comportamentos adequados, mas devido à complexidade do problema de violência é necessária uma abordagem ampla e interdisciplinar abrangendo ações de prevenção e de proteção contra a violência ${ }^{(16)}$.

Além da realização da sistematização dos cuidados de enfermagem a mulheres vítimas de violência doméstica, os enfermeiros que atuam na APS têm a função de capacitar sua equipe ${ }^{(12)}$. Para tanto há que se tenha desenvolvido competências e habilidades específicas para que possam capacitar os demais, o que não é evidente em alguns estudos $(6,7,8,9,10,12)$. Nesse sentido, percebe-se que é importante que o profissional de enfermagem busque estratégias de qualificação para tal.

\section{II) Capacitação dos profissionais de enfermagem frente no reconhecimento da violência doméstica}

O reconhecimento dos enfermeiros da APS obre o que é a violência doméstica e seu importante papel no cuidado frente a ela, seja na anamnese, exame físico e escuta qualificada, ainda apresenta dificuldades causadas pelo medo e insegurança em atender vítimas de violência doméstica, pela falta de treinamento específico e capacitação 
profissional ${ }^{(12)}$. Quanto ao despreparo, ainda existe devido a formação acadêmica dos profissionais de saúde em relação a identificação e no atendimento a pessoas em situações de violência, gerando sentimento de insegurança $^{(17)}$.

Além dos mais, os enfermeiros da APS possuem conhecimentos e interesse sobre a temática da violência contra a mulher, apresentando conhecimentos sobre política de proteção a mulher, notificação compulsória e identificar esse fenômeno como um problema de saúde pública, social, judiciário, econômico e cultural ${ }^{(18)}$. Embora alguns enfermeiros incluem em sua prática profissional um planejamento de ações referentes ao atendimento de mulheres vítimas de violência, relataram que não fizeram cursos de capacitação para atuar na área. havendo desqualificação sobre as leis e decretos que amparam essas mulheres e a necessidade de fortalecer as redes de saúde que recebem esse público ${ }^{(18)}$.

Quanto ao conhecimento dos enfermeiros da atenção primária a respeito da violência contra a mulher, verifica-se um bom resultado dos participantes do que era a violência, perfil epidemiológico, cuidados frente as vítimas, bem como identificar as características do agressor ${ }^{(15)}$. Além do mais, quando são ofertados cursos que capacitam esses profissionais sobre $\mathrm{o}$ atendimento às vítimas de violência, demonstra um ponto satisfatório aos resultados após a sua conclusão que os mesmos se sentem mais preparados e qualificados para atender esse público ${ }^{(17)}$

Os desafios que os serviços de atenção básica enfrentam quanto a violência contra a mulher evidencia que os enfermeiros que estão na linha de frente a assistência deverão estar capacitados para desempar funções e condutas humanizadas, educativas e éticas. No entanto, ainda é observado dentro dos serviços da APS a escassez de preparação dos mesmos no reconhecimento biopsicossocial, necessitando ser desenvolvido educação continua para melhorar e preencher as falhas na assistência interdisciplinar ${ }^{(12)}$.

As limitações que muitos profissionais apresentam é a falta de preparo e formação quanto a abordagem da violência contra a mulher, apresentando falhas desde seu processo de formação consequentemente havendo deficiência não só na assistência frente aos casos de violência, mas o desenvolvimento de competências e habilidades profissionais ${ }^{(19)}$.

É notório que os profissionais de enfermagem que estiveram inseridos nos serviços de atenção primária estejam familiarizados com a temática para que os mesmos possam desempenhar seu papel perante a mulher em situações críticas quanto a violência, bem como desenvolver condutas necessários para que se sintam respaldados e amparos em relação ao encaminhando as redes especializadas e a notificação como 
ferramenta de identificar os casos de violência doméstica.

\section{CONSIDERAÇÕES FINAIS}

Evidenciou-se que as dificuldades que os profissionais de enfermagem têm ao cuidado a mulheres em situações de violência, estão relacionadas a falta de formação qualificada, treinamento e capacitação, sendo identificado sentimento de impotência e despreparo perante as situações vivenciadas na prática clínica, como o encaminhamento aos serviços de referência e a familiaridade com a ficha de notificação compulsória.

Deve-se refletir quais dificuldades essa assistência básica de saúde está enfrentando, proporcionando aos profissionais capacitação, por meio de educação continuada, instrumentalizando-os para identificar e compreender os sentimentos e experiências pelas vítimas, para que fique mais fácil o acolhimento e a qualidade no atendimento.

Sugere-se estudos futuros sobre atuação da equipe de enfermagem na APS sobre vítimas de violência contra crianças e adolescentes e idosos, cuja abordagem é completamente distinta e, esses profissionais deverão estar atentos a esses tipos de sinais de violência.

\section{REFERÊNCIAS}

1. Organização Mundial da Saúde (OMS). Prevenção da violência sexual e da violência pelo parceiro íntimo contra a mulher: ação e produção de evidência [Internet]. São Paulo; 2012. [citado 2021 abr 27]. Disponível em: http://apps.who.int/iris/bitstream/10665/4435 0/3/9789275716359_por.pdf?ua=1

2. Ministério da Saúde (BR), Secretaria de Atenção à Saúde, Departamento de Ações Programáticas Estratégicas. Prevenção e tratamento dos agravos resultantes da violência sexual contra mulheres e adolescentes: norma técnica. Brasília; 2012. (Série Direitos Sexuais e Direitos Reprodutivos; $\mathrm{n}^{\circ}$. 6).

3. Cerqueira D, Bueno S, Lima RSD, Neme C, Ferreira H, Alves PP et al. Atlas da Violência 2020 - Ipea e FBSP. Brasília, DF: Instituto de Pesquisa Econômica Aplicada; 2020. 96 p. Disponível em: https://www.ipea.gov.br/atlasviolencia/downl oad/24/atlas-da-violencia-2020 Acesso em: 21/03/2021.

4. Ministério da Justiça (BR). Lei n. 11.340 de 7 de agosto de 2006. Lei Maria da Penha. Cria mecanismos para coibir a violência doméstica e familiar contra a mulher. Brasília: Ministério da Justiça; 2006. 14p.

5. Miura PO, Silva AC dos S, Pedrosa MMMP, Costa ML, Nobre Filho JN. Violência doméstica ou violência intrafamiliar: análise dos termos. Psicol Soc. 2018;30(0):1-13.

6. Cortes LF, Padoin SMM, Vieira LB, Landerdahl MC, Arboit J. Care for women victims of violence: empowering nurses in the pursuit of gender equity. Rev Gaúcha Enferm. 2015;36(spe):77-84.

7. Visentin F, Becker VieiraL, Trevisan I, Lorenzini E, Franco da SilvaE. Women's primary care nursing in situations of gender violence. invest. educ. enferm [Internet]. 2015Sep.29 [cited 2021Apr.28];33(3). Available from: 
https://revistas.udea.edu.co/index.php/iee/arti cle/view/24465

8. Baraldi ACP, Almeida AM de PGC, Vieira EM. Violência contra a mulher na rede de atenção básica: o que os enfermeiros sabem sobre o problema? Rev. Bras. Saude Mater. Infant. [Internet]. 2012 Sep [cited 2021 Apr 28]; 12(3): 307-318. Available from: http://www.scielo.br/scielo.php?script=sci_art text\&pid=S1519-

$38292012000300010 \& \operatorname{lng}=\mathrm{en}$.

9. Amarijo CLA, Barlem ELD, Acosta DF, Marques SC. Assimilação teórica e prática da violência doméstica: profissionais deenfermagem atendendo vítimas na atenção primária. Rev enferm UERJ, Rio de Janeiro, 2018; 26:e33874

10. Aguiar RS. Nursing care provided to women victims of domestic violence. Rev Enferm do Cent Oeste Min. 2012;3(2):72331.

11. Bardin L. Análise de conteúdo. Lisboa: Edições 70; 2010.

12. Silva VG da, Ribeiro PM. Violência contra as mulheres na prática de enfermeiras da atenção primária à saúde. Esc. Anna Nery [Internet]. 2020 [cited 2021 Apr 28]; 24(4): e20190371. Available from: http://www.scielo.br/scielo.php?script=sci_art text\&pid=S1414-

$81452020000400216 \& \operatorname{lng}=\mathrm{en}$.

13. Silva NNF, Leal SMC, Trentin D, Vargas MADO, Vargas CP, Vieira LB. Atuação dos enfermeiros da atenção básica a mulheres em situação de violência. Enferm em Foco. 2017;8(3):70-4.

14. Netto L de A, Pereira ER, Tavares JMAB, Ferreira D de C, Broca PV. Nursing performance in the conservation of women's health in situations of violence. REME Rev Min Enferm. 2018;22
15. Baraldi ACP, de Almeida AM, Perdoná G, Vieira EM, dos Santos MA. Perception and Attitudes of Physicians and Nurses about Violence against Women. Nurs Res Pract. 2013; 2013:1-7.

16. Hasse M, Vieira EM. Como os profissionais de saúde atendem mulheres em situação de violência? Uma análise triangulada de dados. Saúde debate [Internet]. 2014 Sep [cited 2021 Apr 28]; 38(102): 482493. Available from: http://www.scielo.br/scielo.php?script=sci_art text\&pid=S0103-

$11042014000300482 \& \operatorname{lng}=$ en

17. Pereira Stephanie, Vianna Lucila Amaral Carneiro. Cursos de capacitação em prevenção da violência: o impacto sobre os profissionais do setor da saúde. Rev. esc. enferm. USP [Internet]. 2014 Apr [cited 2021 Apr 28]; 48(2): 315-320.

18. Santos J, Andrade RL de, Reis LAD, Duarte SFP, Conhecimento de enfermeiras em unidades de saúde sobre a assistência à mulher vítima da violência. Rev Baiana Enferm. 2014;28(3):260-70.

19. Marinho PAS, Gonçalves HS. As práticas dos profissionais de saúde em relação à violência de gênero em uma maternidade no Rio de Janeiro. HU Rev. 2016;42(2):97-104.

\section{Autor correspondente:}

Jhuliano Silva Ramos de Souza, Rua da Liberdade, nº72, Vila Betânia, CEP: 37-137090, (35) 991299524. E-mail: jhulianoramoz@hotmail.com

Submissão: 2021-04-29

Aprovado: 2021-05-29 